\title{
Natural aggregation processes as models for architectural material systems
}

\author{
K. Dierichs \& A. Menges \\ Institute for Computational Design, University of Stuttgart, Germany
}

\begin{abstract}
Aggregates are ubiquitous in inanimate natural systems. The capacity of these natural granular systems to continuously adapt to changing environmental influences through cycles of erosion and accretion shows a potential that can be used in architectural design. However, relatively few architectural examples deliberately deploy aggregates in their unbound form. In architecture, granular matter is almost exclusively known in its bound form as an additive in concrete. The aim of this paper is to introduce a practical, methodological and theoretical understanding of the architectural relevance that aggregates in their unbound form can develop. The notion of loose elements as an architectural system is new to architectural design. Architectural systems commonly seek to form a clearly defined assembly of elements, where each part has a place assigned that can be drafted and planned by the designing architect. An aggregate system, however, consists of a loose arrangement of elements, where each part finds its own place and it is the task of the designer to observe and interact with the system. The relevance of these aggregate architectures lies in their ability to continuously adjust to system-external and system-internal parameters. This paper will present the related research into the architectural potential of granular systems. Initially aggregate architecture will be defined and a state of the art overview and analysis of existing examples will be given. Consequently a design development system for aggregate architectures will be given and explained in greater detail. The results are eventually summarized into a conclusive overview and the practical, design-methodological and design-theoretical challenges of an aggregate architecture are outlined.
\end{abstract}

Keywords: aggregate architecture, aggregate architecture development system, natural aggregates, state of the art aggregate architecture. 


\section{Introduction}

Aggregates are ubiquitous in nature. They are embedded in the continuous cycles of erosion and accretion taking place in sedimentary systems, such as deserts, beaches and rivers. The capacity of these natural granular systems lies in their ability to continuously adjust to changing external conditions. Instead of failing or becoming redundant, the material is merely rearranged into a new stable state that is at equilibrium with the changed environmental condition (Siever [1]). In geo-engineering, aggregates are relatively widely researched. One of the pioneering works was conducted by Bagnold [2], who investigated Aeolian sand formations through laboratory and field studies. More recent research also makes an increasingly wide use of numerical and digital experiments (Lanier and Radjaï [3]).

In architecture however, aggregates are mainly known and researched in their bound form as an additive in concrete or earth. Here they merely have the role to strengthen the material composite. Their innate potential of forming adaptive equilibrium states remains largely unexplored. Only few examples in architecture actively use loose aggregates (Hensel and Menges [4]). However, to use the capacity loose granulates are displaying in natural systems can be architecturally relevant, as these systems are never becoming redundant, but instead evolve from one state to the other. These loose aggregate systems are novel to architectural design. Architectural systems are generally developed through assigning specific places to a given material which are planned and drafted by the designer. The designer is in control of the entire assembly. In an aggregate system, each element finds its own place. The role of the designer is merely to observe and interact with the system. He becomes an inter-actor with a continuously changing arrangement of elements (Hensel and Menges [5]).

The aim of the paper is to formulate an initial definition of an aggregate architecture. This definition will subsequently be used to compose a state of the art overview of existing projects falling into this notion. These projects then allow for giving a first indication of a possible development system of aggregate architectures. Eventually the theoretical, methodological and practical consequences are indicated and an outlook over further areas of research is given.

\section{Aggregate architectures}

\subsection{Definition of aggregate architectures}

Aggregates are defined as large amounts of elements in loose contact (Nedderman [6]). They can range from powders with a scale range of less than 100 ìm in diameter over granular solids with ranges from 100 ìm to 3000 ìm to broken solids where most particles are larger than $3 \mathrm{~mm}$ (Duran [7]).

An architectural material system can be defined as a material system that is capable of performing architecturally relevant tasks. A material system is in its geometry and topology informed by the self-organizational capabilities of a 
material and by environmental criteria. In an architectural material system these are typically criteria of spatial organization, climatic modulation, structural performance, relationships to the location as well as those of performance in a social context and programme (Hensel and Menges [8]).

An aggregate architecture can consequently be defined as an architectural material system consisting of aggregates. An arrangement of large numbers of loose elements is left to self-organize. The critical aspect of this specific material system lies in the second defining aspect of an architectural material system, which is the information of the system with typically architectural criteria. The problematic lies in the fact, that the actual manipulation of the system to match certain criteria is not possible. What is possible, however, is to choreograph the system in its evolving states. One might say that these criteria of architectural performance can merely be observed and possibly triggered either through the design of the individual elements or through the composition and modulation of the overall system.

As an initial definition, aggregate architectures can thus be described as large arrangements of loose elements that are continuously observed and modulated by the architect on the particle- or system-level to perform one or more typically architectural tasks. At this point it needs to be stated, that in certain scientific schools of thought, there is a discussion as to whether aggregates can be called systems or whether they rather are their direct opposite (Hensel and Menges [9]). However, we are in this paper adopting the natural scientific terminology that consistently calls aggregates, systems' (Duran [10]).

Going back to the natural model of sedimentary granular systems, a clear delineation is now needed that identifies the similarities and differences between the natural and the architectural model. The correspondence between the two models certainly lies in the physical definition of the system as an arrangement of loose elements and its consequent ability to adapt into continuously changing equilibrium states. The difference however lies in the term that a designing

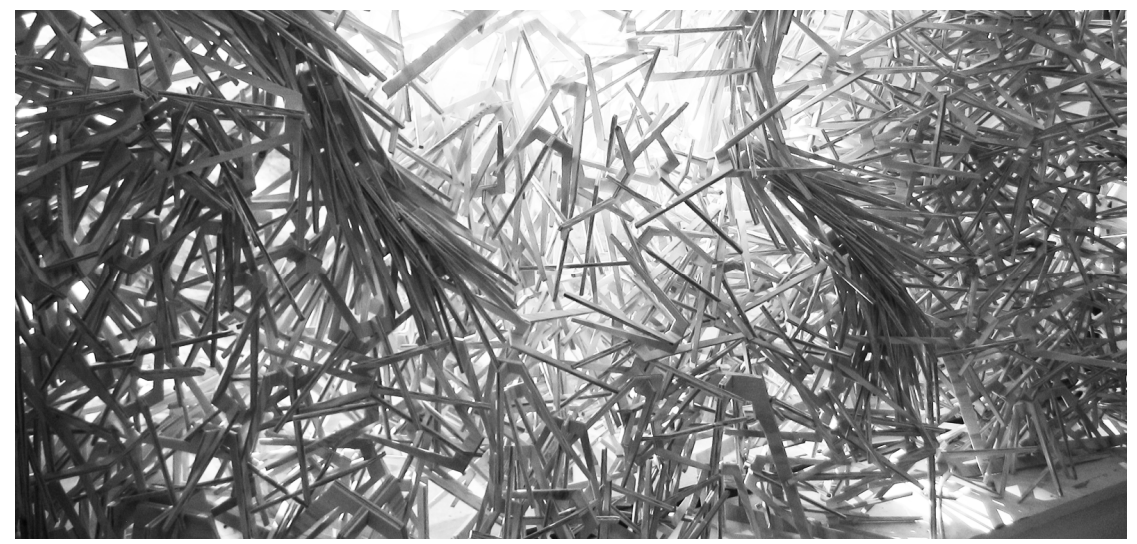

Figure 1: Aggregate Architecture 01, A. Hawkins and C. Newell, Tutors: M. Hensel and A. Menges, image credit: A. Menges. 


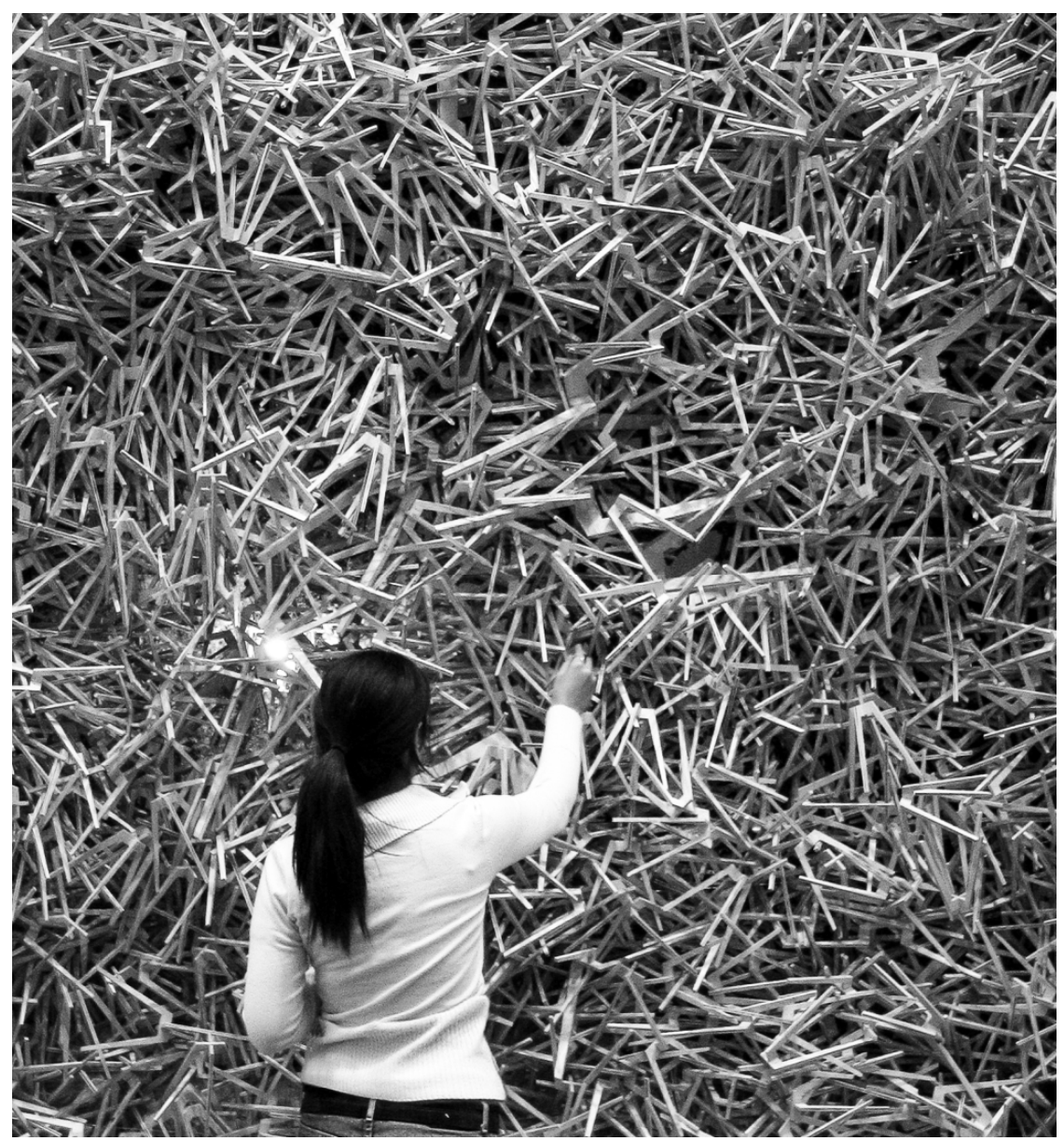

Figure 2: Aggregate Architecture 02, A. Hawkins and C. Newell, Tutors: M. Hensel and A. Menges, image credit: A. Menges.

architect consciously observes and modulates the system on the particle- or system-level to perform typically architectural tasks. This level of conscious interaction with the system and induction of possible effects does not take place in natural aggregation processes. The model of aggregate architectures is therefore deploying the capacities of adaptation of natural granulates, yet it adds a second level of conscious architectural modulation to the system.

\subsection{State of the art overview of aggregate architectures}

The following will give an overview of projects and applications, which might already be considered aggregate architectures. They are selected and composed with respect to the definition deduced in the previous chapter. What is striking is

WIT Transactions on Ecology and the Environment, Vol 138, (c) 2010 WIT Press www.witpress.com, ISSN 1743-3541 (on-line) 
that they are not necessarily produced in the field of architecture itself, but come from different fields that deal with granulates for one or the other reason.

There are five main fields or categories where aggregate architectures can be found. Each of them will be introduced describing the most relevant architectural applications of loose granulates in that specific field. It is crucial to note at this point, that not in all cases the granular arrangements are seen as architectural by the makers themselves. Yet they are forms of aggregate architectures, given the above description is applied.

Category 1 is the field of Building Physics. Granulates are used as sound and temperature insulating fillers (Hausladen et al. [11]). Here, the architectural performance is solely on the level of climatic modulation.

Category 2 is taken from the field of Building Construction. Sand is in this case used as a flexible mould for the assembly of elements (Treib [12], Marks [13]). The architectural relevance of the aggregate lies in this case in its supportive role as a reusable and flexible construction material.

Category 3 is the area of geo-engineering. In this field especially granular matter is processed in vast amounts, yet very seldom one can identify an architectural impact of these interventions (Trummer [14]). There are however some applications that combine an architectural role of a respective sedimentary granular system and its landscape engineering implications (Sherzad [15], Hensel et al. [16]).

Category 4 encompasses the field of Earth Architecture. A wide variety of earth construction techniques can be found here, yet the aggregate is mostly embedded in a binding matrix. Two techniques however use loose or semi-loose granular matter. These are on the one hand so-called 'fill-ins', where loose aggregate is poured into a containing body and works as an insulating and stabilising sub-system within the overall architectural system (Houben and Guillaud [17]). This same technique can also be found in non-vernacular architectures (Herzog and de Meuron [18]). The second application is horizontal dug-outs. These are carved into relatively loose soil and show stable archformations which are characteristic of aggregates (Houben and Guillaud [19]).

Category 5 is the relatively largest area of aggregate exploration. Furthermore aggregates are here consciously used for their properties in their unbound form. One might state that in this field, the notion of an actual aggregate architecture is most strongly developed. Yet no consistent approach to the subject exists even in this area and the projects show the problem, that a coherent scientific framework for aggregate architectures does not exist. At the Institute for Lightweight Structures under Frei Otto, several form-finding experiments with sand were conducted, mainly testing the formations of arches and caves, pile and funnel relationships as well as erosion patterns (Gaß and Otto [20]). Another very resourceful area of aggregate research has been opened up by the Swiss doctor and natural scientist Hans Jenny (1904-1972). He studied the formation of aggregate patterns under the influences of vibration and conducted a large amount of physical experiments with this basic set-up, such as the Chladni Figures (Jenny [21]). Applications within architecture have been considered and range from the use of these techniques in seismic areas to systems driven by 


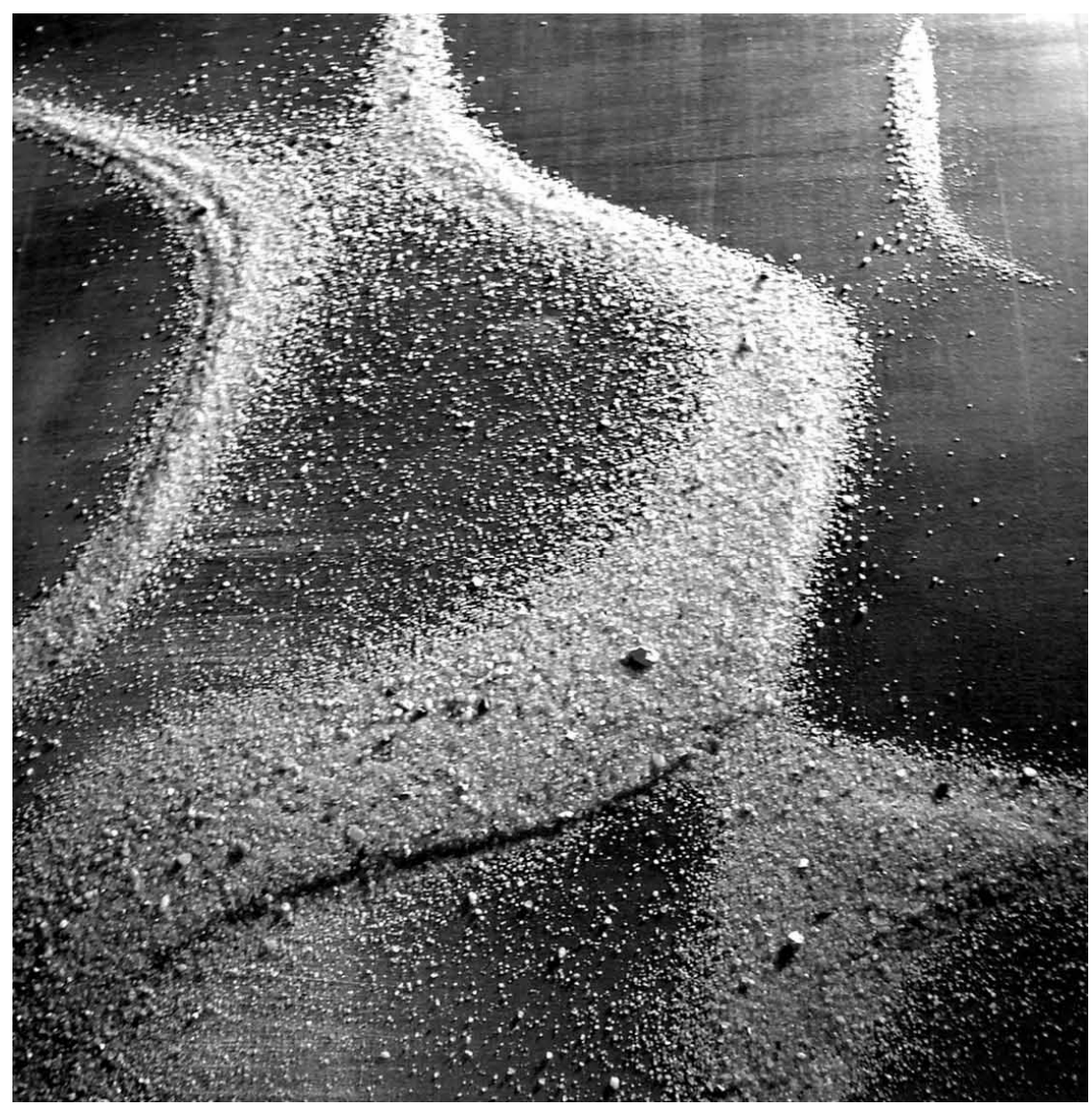

Figure 3: Aggregate architecture 03, K. Dierichs, Tutors: M. Hensel, A. Menges and M. Weinstock, image credit: K. Dierichs.

artificial vibration (Hensel and Menges [22]). Relating to the work conducted under Frei Otto, several experiments have been conducted in recent years that develop the idea of aggregates as an architectural system further. They can be distinguished into two main groups, one working with natural and one working with artificial aggregates. Projects based on natural aggregates bear a close relationship to applications found in Category 3, the aggregate architectures found in geo-engineering. They modulate natural sediments through specifically designed structures or strategic reorganization of the granular material (Fallaha [23], Takahashi [24], Hensel et al. [25]). The second group of projects uses artificial particles. These are designed to specific effects, such as an increase in friction between the elements on a micro- and macro-level. The resulting structures perform on several levels. Arches can be poured and subsequently self-stabilized over a pneumatic formwork. The structures are also studied for light transmission and water-flow, i.e. for criteria of modulating 


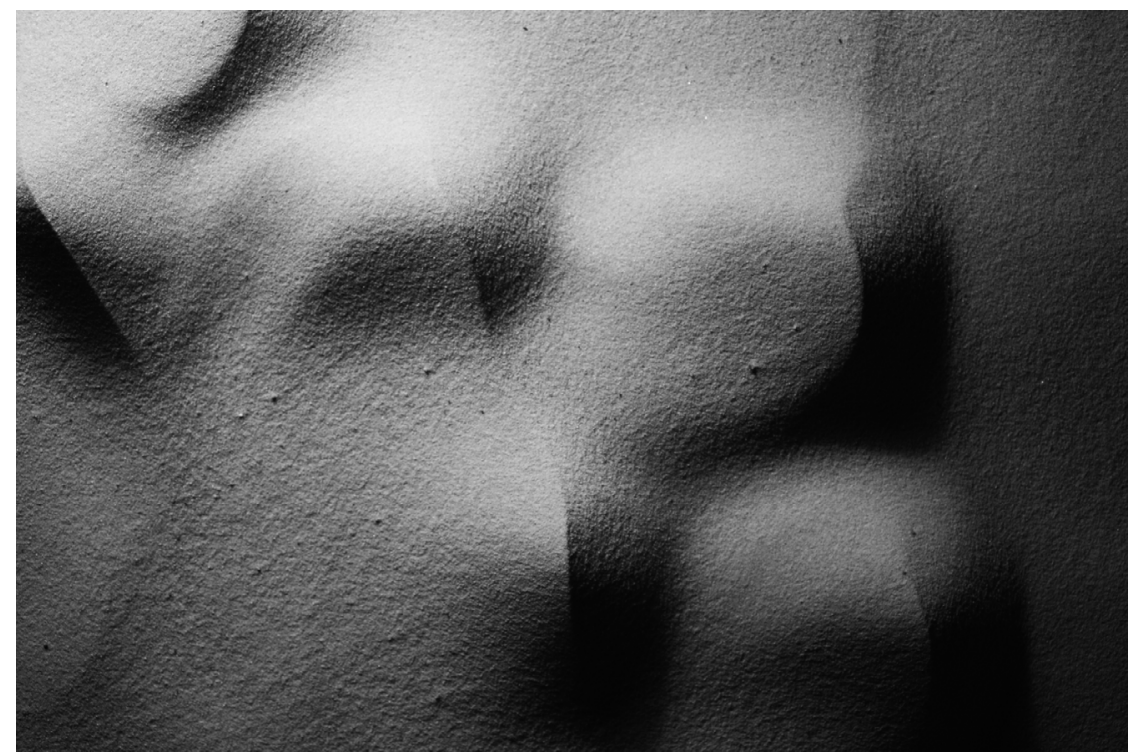

Figure 4: Aggregate architecture 04, K. Dierichs, Tutors: M. Hensel, A. Menges and M. Weinstock, image credit: K. Dierichs

environmental parameters. Larger scale applications with an approximate arch height of 1.50 metres have been equally tested in this category of designed aggregates (Hawkins and Newell [26], Matsuda [27], Hensel et al. [28]).

This state of the art overview presented shows that there are few examples of architectural systems that can be described as aggregate architectures. An analysis of the collected results shows several prominent aspects. First, almost only natural aggregates are used except for very few examples. Second, gravity is the main force effective on the granular systems and is in some cases combined with wind or airstreams. Third, spatial formations in aggregates are either semi-enclosures or arch- and cave formations. Fourth, there is quite a range of applications that use the granular capacity to modulate environmental conditions, such as sound, heat and cold, wind, light or rainwater. Here, one can also find the integration of several combined architectural performances in a single aggregate arrangement. Overall one can state from this analytical overview that a coherent scientific framework of development does not exist for aggregate architectures and the potential range of this specific material system is consequently not explored.

\subsection{Development system for aggregate architectures}

In order to develop a consistent approach to an aggregate architecture, a development system needs to be laid out that allows for researching and subsequently embedding specific aspects of the overall granular system. 
The suggested development system consists of two main categories. The first one deals with the questions around how the material system is set up and maintained, the second one serves to evaluate how the system performs on an architectural level. Both of them have a set of sub-categories. The following gives a listed explanation of both the two main- and their respective subcategories.

[i] The first main category of aspects belonging to the set-up and maintaining of the system is formulated to encompass criteria that allow for defining the aggregate system itself, its boundaries and forces working on the system, as well as the process of the architect interacting with the system. The sub-categories can be defined as follows: [i.i] Natural or artificial particles: This sub-category deals with the provenance of the particles themselves, i.e. whether they are naturally sourced or artificially made. In case they are artificially made, this sub-category also deals with material, geometry and manufacturing of these individual elements. [i.ii] System grading: The system grading describes the particle size distribution in a granulate. This can have quite strong effects on the overall system behaviour and can thus be deployed strategically in the process of design. [i.iii] System size: The system-size can be defined through mainly two variables, namely either the amount of particles or the overall volume that the granulate is able to fill. [i.iv] System boundary: The system boundary describes on the macro-level the geometric constraints the aggregate system might have, such as a container, a wall or a ground surface. On the micro-level the roughness of the respective surface is very relevant in order to determine the friction between the particles and their boundary. [i.v] Effective forces on the system: the system can be exposed to a set of forces working on it. These are gravity, external loads, airstreams or winds, vibrations and in some cases also temperature can be a relevant testing parameter. [i.vi] Process of casting and interacting with the system: In this sub-category, the exact casting method, such as the pouring speed and pouring outlets, is tested. In addition, the continuous subsequent interactions of the designing architect are classified and tested in this section.

[ii] The second main category of aspects evaluating the architectural performance of the system as it were helps to observe the aggregate behaviour with respect to specific aspects that are architecturally relevant. For this main category, the same parameters describing an architectural material system have been used, as those that have been introduced in chapter 2.1. In the following paragraph, the individual sub-categories are listed and explained in more detail. [ii.i] Spatial configuration: This sub-category is aimed at analyzing spaces resulting from processes of aggregation. These can be either fully or semienclosed. Those spaces can be described through their spatial dimensions and the interconnections between individual spaces. [ii.ii] Environmental modulation: In this sub-category, the aggregation is evaluated with regards to its capacity to specifically modulate certain environmental influences. These can be light, sound, airflow, heat or rainwater. [ii.iii] Structural performance: The structural performance of a certain aggregate configuration can be tested with regards to the system's loading under its self-weight as well as a set of externally applied loads. [ii.iv] Programmatic use: Programme in architecture describes how spaces 
are used. This can be a time-dependent process and is closely related to the system's environmental modulation and spatial configuration. [ii.v] Relationship to context: This sub-category is aimed at investigating how the granular system is embedded within its context.

The aim of this design development system is to offer a coherent research framework of the aggregate architectural model. Specific, focused aspects can be worked on in smaller steps and subsequently be embedded within the overall system. Increasingly questions of the exact interrelation between the two main categories, i.e. the system set-up and its architectural performance, will be placed to allow for a more and more reliable calibration of the granular system to attain specific architectural effects.

\section{Conclusion}

Aggregate architecture has been defined as a system consisting of large numbers of loose elements that are observed and modulated by the architect on the particle- or system-level to perform one or more typically architectural tasks. A cross-disciplinary state of the art overview has been given. This shows that a consistent and comprehensive approach to an aggregate architecture does not exist, respectively is only in its beginnings. The existing projects do not as of yet deploy the full range of potential aggregate behaviours as observed in natural granular substances and the consequent architectural implications thereof. A possible outline for a development system of an aggregate architecture has been suggested.

Aggregate architectures are novel to the concept of architectural systems on a practical, design-methodological and design-theoretical level. The notion of an arrangement of loose elements questions the necessity to perceive of architecture as a clearly defined permanent entity. Similar to the constant adaptation processes in natural granular masses, aggregate architectures can only be understood as transitory states and thus providing a challenge to the architect to predict their patterns of behaviour reliably, instead of allowing him to clearly define the specific location of elements and their permanent overall assembly.

\section{References}

[1] Siever, R., Sand: Ein Archiv der Erdgeschichte, Spektrum der Wissenschaft: Heidelberg, pp. 185-190, 1988, German Edition 1989.

[2] Bagnold, R.A., The Physics of Blown Sand and Desert Dunes, Dover Publications: Mineola, 1954, $2^{\text {nd }}$ edition 2005.

[3] Lanier, J. \& Radjaï, F., Experimental and Numerical Analysis of Local Variables in Granular Materials (Chapter 1). Micromechanics of Granular Materials, eds. B. Cambou, M. Jean \& F. Radjaï, ISTE Ltd. and John Wiley \& Sons Ltd.: London and Hoboken, pp. 1-49, 2009.

[4] Hensel, M. \& Menges, A., Aggregates. Versatility and Vicissitude, AD Architectural Design, Profile No. 192, 78 (2), pp. 80-87, March/April 2008. 
[5] Hensel, M. \& Menges, A., Materialsysteme 05: Aggregate. Form Follows Performance: Zur Wechselwirkung von Material, Struktur, Umwelt, Arch+, 188, pp. 76-85, Juli 2008.

[6] Nedderman, R.M., Statics and Kinematics of Granular Materials, Cambridge University Press: Cambridge, p. 1, 1992, $2^{\text {nd }}$ edition 2005.

[7] Duran, J., Sands, Powders and Grains: An Introduction to the Physics of Granular Materials, Partially ordered systems, Springer: New York, pp. 25-26, 2000.

[8] Hensel, M. \& Menges, A., Material Systems - Proto-Architectures (Part 2). Morphoecologies, eds. M. Hensel \& A. Menges, AA Publications: London, pp. 62-67, 2006.

[9] Hensel, M. \& Menges, A., Material Systems - Proto-Architectures (Part 2). Morphoecologies, eds. M. Hensel \& A. Menges, AA Publications: London, p. 64, 2006.

[10] Duran, J., Sands, Powders, and Grains: An Introduction to the Physics of Granular Materials, Partially Ordered Systems, Springer: New York, 2000.

[11] Hausladen, G., de Saldanha, M. \& Liedl, P., Climaskin: Konzepte für Gebäudehüllen, die mit weniger Energie mehr leisten, Callway: München, pp. 122-123, 2006.

[12] Treib, M., Space Calculated in Seconds: The Philips Pavilion, Le Corbusier, Edgar Varèse, Princeton University Press: Princeton and Chichester, pp. 52-97, 1996.

[13] Marks, D., Storm Watershed. Environmental Tectonics: Forming Climatic Change, AA Agendas No. 6, ed. S. Hardy, AA Publications: London, pp. 60-63, 2008.

[14] Trummer, P., Engineering Ecologies. Versatility and Vicissitude, AD Architectural Design, Profile No. 192, 78 (2), pp. 96-101, March/April 2008.

[15] Sherzad, M. I., Built Form and Aeolian Sand Deposits in the Algerian Sahara, Doctoral Thesis Oxford Brookes University: Oxford, 1996.

[16] Hensel, M., Menges, A. \& Weinstock, M., Emergent Technologies and Design: Towards a biological paradigm for architecture, Routledge: Abingdon, pp. 227-236, 2010.

[17] Houben, H. \& Guillaud, H., Earth Construction: A Comprehensive Guide, Intermediate Technology Publications: London, pp. 170-171, 1989, English edition 1994.

[18] Herzog, J. \& de Meuron, P., Herzog \& de Meuron 1993 1997. El Croquis, 84, pp. 182-191, 1997.

[19] Houben, H. \& Guillaud, H., Earth Construction: A Comprehensive Guide, Intermediate Technology Publications: London, pp. 166-167, 1989, English edition 1994.

[20] Gaß, S. \& Otto, F. eds., Experimente / Experiments, Form - Kraft - Masse 5 / Form - Force - Mass 5. Mitteilungen des Instituts für leichte Flächentragwerke (IL) Universität Stuttgart Nr. 25 / Information of the Institute for Lightweight Structures (IL) University of Stuttgart No. 25, 
Karl Krämer Verlag: Stuttgart, pp. 2.6, 2.15, 2.22, 2.34, 2.35, 2.88, 2.89, 2.90, 2.91, 2.92, 2.93, 1990.

[21] Jenny, H., Cymatics: A Study of Wave Phenomena and Vibration, Macromedia Publishers: Newmarket NH, 2001.

[22] Hensel, M. \& Menges, A., Aggregates. Versatility and Vicissitude, AD Architectural Design, Profile No. 192, 78 (2), p. 83, March/April 2008.

[23] Fallaha, H., Adaptive Pneumatic Shelters 2003-2004 (Chapter 2.15). Morpho-Ecologies, eds. M. Hensel \& A. Menges, AA Publications: London, pp. 232-241, 2006.

[24] Takahashi, G., Aggregates 03 2005-2006 (Chapter 2.20). MorphoEcologies, eds. M. Hensel \& A. Menges, AA Publications: London, pp. 286-295, 2006.

[25] Hensel, M., Menges, A. \& Weinstock, M., Emergent Technologies and Design: Towards a biological paradigm for architecture, Routledge: Abingdon, pp. 227-236, 2010.

[26] Hawkins, A. \& Newell, K., Aggregates 022004 (Chapter 2.19). MorphoEcologies, eds. M. Hensel \& A. Menges, AA Publications: London, pp. 274 283, 2006.

[27] Matsuda, E., Aggregates 01 2003-2004 (Chapter 2.18). Morpho-Ecologies, eds. M. Hensel \& A. Menges, AA Publications: London, pp. 262-271, 2006.

[28] Hensel, M., Menges, A. \& Weinstock, M., Emergent Technologies and Design: Towards a biological paradigm for architecture, Routledge: Abingdon, pp. 236-241, 2010. 